\title{
Boussinesq's problem of viscoelasticity
}

\author{
Detlef Wolf \\ Institute of Planetology, University of Münster, Wilhelm-Klemm-Str. 10, D-4400 Münster, FRG.
}

\begin{abstract}
We consider an isochemical, isentropic, incompressible fluid halfspace and study quasistatic viscoelastic perturbations, induced by two-dimensional (2D) surface loads, of a hydrostatic initial state. In view of the regional or local scale required for deformations of planets to be amenable to the half-space approximation, the model is assumed to be externally gravitating. We derive analytic solutions for the displacement and incremental stress components and study several approximations to the expressions. Particular emphasis is placed on discriminating between the material and local incremental stresses. Based on this distinction, deeper insight is gained into the physical significance of the solution.
\end{abstract}

Terra Nova, 3, 401-407

\section{INTRODUCTION}

Detailed investigations into the elastostatic deformation of a plane half-space subject to either displacement or traction boundary conditions were carried out by Boussinesq over a century ago and are summarized in his 1885 monograph. Today, the basic problem is usually referred to as Boussinesq's problem, although in part of his work Boussinesq was preceded by Lamé \& Clapeyron (1831) and Cerruti (1882). Since the publication of Boussinesq's monogaph, numerous authors have written on particular aspects of Boussinesq's problem (e.g. Lamb, 1902; Terazawa, 1916; Love, 1929; Harding and Sneddon, 1945; Sneddon, 1946; Farrell, 1972).

A common feature of most work on Boussinesq's problem is that the unperturbed half-space is regarded as unstressed. As far as the model is applied to study the regional or local deformation of planets, this assumption clearly cannot be satisfied. Effects due to a planet's initial stress are, however, small for elastic perturbations whose lateral wavelength is in sufficiently short for the application of the half-space approximation (Cathles, 1975, pp. 35-39).

More significant is the influence of the initial stress for viscoelastic perturba- tions. Such problems were extensively studied by Biot and are reviewed in a monograph (Biot, 1965). Closely related to the present study is Biot's analysis of the quasistatic viscoelastic deformation of an initially hydrostatic half-space (Biot, 1959), in which effects due to the initial stress are accounted for by a separate term included in the incremental equilibrium equation. In general, Biot preferred a formal treatment of the problem, in which the physical significance of the modifications associated with the initial stress was not fully discussed.

Unfortunately, Biot's work has not received much attention from geophysical researchers. This disregard is evident in several subsequent studies of the viscoelastic Boussinesq's problem, in which the initial stress is neglected (e.g. Peltier, 1974; Cathles, 1975, pp. 57-59).

Research on the viscoelastic Boussinesq's problem was resumed by Nakiboglu and Lambeck (1982) and Wolf (1985a, 1985b, 1985c), who studied the response due to surface loading. Whereas Nakiboglu and Lambeck (1982) accounted for the influence of the initial stress by an ad hoc modification of the incremental boundary condition, Wolf included such effects in the incremental equilibrium equation. The distinction between 'viscoelastic' and 'total' pertur- bation stresses then allowed Wolf to reduce the incremental field equations formally to those valid in the absence of initial stress, which could be solved using elementary methods.

In retrospect, Wolf's (1985a) method of accounting for the initial stress is seen to be very similar to that used by Biot (1959), although Wolf was not aware of Biot's publication at that time. As in Biot's study, the significance of the modifications associated with the initial stress was not fully recognized by Wolf $(1985 a, b)$. This, in particular, applies to the physical meaning of the two kinds of incremental stress employed in Wolf's analysis, which was not adequately discussed.

Recently, the theory of viscoclastodynamics for fluids in a state of hydrostatic initial stress has been reviewed (Wolf, 1991). In particular, rigorous deductions were given for the incremental field equations and continuity conditions and of the asymptotic approximations of the equations for short and long times after the onset of the perturbations. Special emphasis was placed on the distinctions between the Lagrangian and Eulerian kinematic formulations of the equations and between the material and local increments of the field quantities. Based on this, it was possible to interpret the short- and long-time asymptotic equations as the incremental field equations and continuity conditions of elastodynamics and of fluid dynamics, respectively.

In view of the progress achieved in our understanding of the theory of viscoelastodynamics for fluids in a state of hydrostatic initial stress, a re-examination of the viscoelastic Boussinesq's problem within this improved theoretical framework appears to be justified. In the first place, such a re-examination is intended to clarify the physical interpretation of previous solutions to the problem. However, it should also serve as a guide for the physically correct treatment of more complicated problems. 
In agreement with the heuristic character of the present study, the model to be analyzed is kept as simple as possible. We therefore consider the viscoelastic Boussinesq's problem using an isochemical, isentropic, incompressible half-space deformed by a $2 D$, harmonic surface load. Since effects due to the perturbation of the gravity field are small for local or regional deformations of planets (Cathles, 1975, pp. 72-83), the halt-space is assumed to be externally gravitating. In section 2 , the relevant incremental field equations and boundary conditions and their Laplace transforms are collected. The equations are solved in section 3 by means of Love's strain function and inverse Laplace transformation. Section 4 gives a discussion of the solution. Special care will be taken to discriminate between material and local incremental stresses. This distinction will prove necessary for a physically correct interpretation of the solution and its approximations.

\section{FIELD EQUATIONS AND BOUNDARY CONDITIONS}

The present study is concerned with Cartesian tensor fields. For brevity, we use for the fields the indical notation and summation convention stipulating that the index subscripts $i, j, k$ range over $1,2,3$ and repeated indices imply summation. Note that the summation convention will later be suspended for the label subscripts $x, y, z$. We also employ the differentiation convention, i.e. index and label subscripts preceded by a comma denote partial differentiation with respect to the coordinate direction indicated by the subscript.

We assume now that the current state of a fluid at the time $t \in[0, \infty)$ represents a small increment with respect to a hydrostatic initial state at the time $t=0$. Further, we take as the spatial argument the initial particle position, $X \in \mathscr{V}^{(0)}$, with $\vartheta^{(a)}$ the (open) region initially occupied by the fluid. This is commonly referred to as the Lagrangian kinematic formulation. The perturbation of an arbitrary initial field, $f_{i j}^{(U)}(\mathbf{X})$, can then be alternatively described in terms of the material incremental field, $f^{(\delta)}(X, t)$, observed at the particle initially at $X$, or in terms of the local incremental field, $f_{i, \ldots}^{(\Delta)}(X, t)$, observed at the initial position, $X$. The material and local incremental fields are related by

$f_{i j \ldots}^{(\delta)}(\mathbf{X}, t)=f_{i j \ldots}^{(\Delta)}(\mathbf{X}, t)+f_{i j \ldots, k}^{(0)}(\mathbf{X}) u_{k}(\mathbf{X}, t)$,

where $u_{i}(\mathbf{X}, t)$ is the particle displacement and $f_{i j \ldots k}^{(0)}(\mathbf{X}) u_{k}(\mathbf{X}, t)$ the advective incremental field (for further details cf. Dahlen, 1974; Grafarend, 1982; Wolf, 1991).

For the present study, we assume that the fluid is isochemical, isentropic, non-rotating, externally gravitating and subject only to gravitational volume forces. On these assumptions, the equations governing the initial state (e.g. Wolf, 1991) reduce to

$-p_{, i}^{(0)}+\rho^{(0)} g_{i}=0$,

$\rho^{(0)} p_{, i}^{(0)}=k \rho_{, i}^{(0)}$,

where $g_{i}$ is the gravitational force per unit mass, $k$ the (isentropic) fluid bulk modulus, $p^{(0)}$ the initial mechanical pressure, $\rho^{(0)}$ the initial mass density and the argument $\mathbf{X}$ has been suppressed. Equations (2) and (3) are referred to as the initial equilibrium equation and the initial state equation. With $g_{i}$ and $k$ prescribed fields, (2) and (3) constitute the system of initial field equations to be satisfied by $p^{(0)}$ and $\rho^{(0)}$ for all $\mathrm{X} \in \mathcal{V}^{(0)}$. $x \in \mathscr{V}^{(0)}$.

Assuming now that the fluid undergoes quasistatic viscoelastic perturbations, the following equations apply (e.g. Wolf, 1991):

$$
\begin{aligned}
t_{i, j}^{(\delta)} & +\left(p_{, j}^{(0)} u_{j}\right), i_{i}-g_{i}\left(\rho^{(0)} u_{j}\right), j=0 \\
t_{i j}^{(\delta)}= & \int_{0}^{1}\left[m_{1}\left(t-t^{\prime}\right)-\frac{2}{3} m_{2}\left(t-t^{\prime}\right)\right] \partial_{l^{\prime}} u_{k, k}\left(t^{\prime}\right) \delta_{i j} d t^{\prime} \\
& +\int_{0}^{t} m_{2}\left(t-t^{\prime}\right) \partial_{t^{\prime}}\left[u_{j, i}\left(t^{\prime}\right)+u_{j, i}\left(t^{\prime}\right)\right] d t^{\prime}
\end{aligned}
$$

where $m_{1}\left(t-t^{\prime}\right)$ and $m_{2}\left(t-t^{\prime}\right)$ are the bulk and shear relaxation functions, $t_{i j}^{(\delta)}$ is the material incremental Cauchy stress, $\delta_{i j}$ the Kronecker symbol, $\partial_{i}$, the partial derivative operator with respect to $t^{\prime}$ and the arguments $X$ and $t$ have been suppressed. Equations (4) and (5) are referred to as the incremental equation of motion and the incremental constitutive equation of viscoelasticity. With $m_{1}\left(t-t^{\prime}\right)$ and $m_{2}\left(t-t^{\prime}\right)$ prescribed fields and $p^{(0)}$ and $\rho^{(0)}$ obtained by solving
(2) and (3), equations (4) and (5) constitute the system of incremental field equations of quasistatic viscoelastodynamics to be satisfied by $t_{i j}^{(\delta)}$ and $u_{i}$ for all $\mathbf{X} \in V^{(0)}$ and $t \in[0, \infty)$.

To simplify the problem further, we assume that the fluid is incompressible. In the initial state, we thus have

$k \rightarrow \infty$,

$\rho_{, l}^{(0)} \rightarrow 0$

With (6) and (7), the initial field equations, (2) and (3), reduce to a single equation

$-p_{, i}^{(0)}+\rho g_{i}=0$,

where $\rho \geq 0$ is now a parameter. On the boundary $\mathcal{A}^{(0)}$ of the region $v^{(0)}$ initially occupied by the fluid, $p^{(0)}$ must satisfy conditions to be prescribed. We assume here that the boundary of the fluid is initially a free surface and therefore normal to $g_{i}$. With $n_{i}$ the unit vector in the direction of $g_{i}$ and the notation

$\left[f_{i j \ldots}\right]_{7+}=\lim _{\boldsymbol{\epsilon} \rightarrow f_{+} \ldots} f_{j}(\mathbf{X}+\epsilon \mathbf{n})$,

the initial boundary condition takes the form (e.g. Wolf, 1991)

$\left[p^{(0)}\right]_{+}=0$,

where $\mathbf{X} \in \mathcal{S}^{(0)}$. Note that, by (8) and (10), the conditions $p^{(0)}=0$ and $\rho g_{i} \doteq 0$ are equivalent for all $X \in \mathcal{V}^{(0)}$. The fluid we are concerned with is therefore unstressed in the initial state only if it is non-gravitating.

On the assumption of incompressibility, it follows for the incremental state that

$m_{1}\left(t-t^{\prime}\right) \rightarrow \infty$,

$u_{i, i} \rightarrow 0$.

We also assume that $m_{2}$ is spatially homogeneous. Introducing $m=m_{2}$ for brevity, we thus require

$m_{. i}\left(t-t^{\prime}\right)=0$.

In view of (7), (11) and (12), the incremental field equations, (4) and (5), are replaced by

$t_{i j, !}^{(\delta)}+\left(p_{,}^{(0)} u_{i}\right)_{, i}=0$,
$u_{i, i}=0$,

$t_{i j}^{(\delta)}=-p^{(\delta)} \delta_{i j}+\int_{0}^{1} m\left(t-t^{\prime}\right)$

$\times \partial_{t^{\prime}}\left[u_{i, j}\left(t^{\prime}\right)+u_{i, i}\left(t^{\prime}\right)\right] d t^{\prime}$, 
where we have introduced

$p^{(\delta)}=-\int_{0}^{t} \lim _{\substack{u_{u_{1}} \rightarrow 0 \\ m_{1}\left(t^{\prime}-\ell^{\prime}\right) \rightarrow \infty}} m_{1}\left(t-t^{\prime}\right) \partial_{t^{\prime}} u_{i, j}\left(t^{\prime}\right) d t^{\prime}$.

For all $X \in \mathscr{A}^{(0)}$, the solution to the incremental field equations must satisfy prescribed conditions. Here, we are only concerned with perturbations due to surface loads. Employing the notation introduced in (9), the incremental boundary condition takes the form (e.g. Wolf, 1991)

$\left[n_{j} t_{i j}^{(\delta)}\right]_{+}=-n_{i} q$,

where $q$ is the prescribed incremental load pressure and $X \in \mathscr{A}^{(0)}$.

The general solution to (14)-(16) can be derived by means of elementary methods if $p^{(\delta)}$ and $t_{i j}^{(\delta)}$ are expressed in terms of the associated local increments. Since $t_{i j}^{(0)}=-p^{(0)} \delta_{i j}$, it follows from (1) that

$p^{(\delta)}=p^{(1)}+p_{. i}^{(0)} u_{i}$

$t_{i j}^{(\delta)}=t_{i j}^{(\Delta)}-p_{, k}^{(0)} u_{k} \delta_{i j}$.

Substitution of (19) and (20) into (14)(16) and (18) gives

$t_{i, j}^{(\Delta)}=0$,

$u_{i, i}=0$,

$t_{i j}^{(\Delta)}=-p^{(\Delta)} \delta_{i j}+\int_{0}^{t} m\left(t-t^{\prime}\right)$

$$
\times \partial_{t^{\prime}}\left[u_{i, j}\left(t^{\prime}\right)+u_{j, i}\left(t^{\prime}\right)\right] d t^{\prime},
$$

$\left[n_{j} i_{i j}^{(\Delta)}-n_{i} p^{(0)} u_{j}\right]_{+}=-n_{i} q$.

Note that the incremental field equations, (21)-(23), no longer depend on $p_{i,}^{(0)}$, and thus in particular agree with the ordinary field equations valid in the absence of initial stress. However, effects due to the initial stress enter through the incremental boundary condition, (24), which explicitly involves $p_{, i}^{(0)}$.

Solutions to (21)-(23) subject to (24) will be obtained by means of the Laplace transforms of the equations. The Laplace transform, $\mathscr{L}[f(t)]$, of a function $f(t)$ is defined by

$\mathscr{L}[f(t)]=\bar{f}(s)=\int_{0}^{*} f(t) e^{-s t} d t$,

where the transform variable, $s$, will be restricted to real values. For the convergence of the integral in (25) for $s$ larger than some value $s_{0}$ it is sufficient that $f(t)$ be piecewise continuous for all $t \in[0, \infty)$ and of exponential order as $t \rightarrow \infty$. Elementary consequences of (25) are (e.g. LePage, 1961, pp. 285-328)

$\mathscr{L}\left[\partial_{t} f(t)\right]=s \vec{f}(s)-f(0+)$,

$\mathscr{L}\left[\int_{0}^{t} f\left(t-t^{\prime}\right) g\left(t^{\prime}\right) d t^{\prime}\right]=\tilde{f}(s) \tilde{g}(s)$.

We will also need

$\mathscr{L}\left[H(t) e^{-s^{\prime} t}\right]=\frac{1}{s+s^{\prime}}$,

where $H(t)$ is the unit step function, here formally defined by

$H(t)=\lim _{\epsilon \rightarrow 0}\left\{\begin{array}{l}\frac{t}{\epsilon}, 0 \leq t \leq \epsilon \\ 1, \epsilon<t<\infty\end{array}\right.$

Using (25)-(27) and assuming that $u_{i, j}$ vanishes at $t=0+$, we obtain from (21)-(24) the Laplace-transformed incremental field equations and boundary condition:

$\tilde{t}_{i j, l}^{(\Delta)}=0$,

$\tilde{u}_{i, i}=0$,

$\tilde{t}_{i j}^{(\Delta)}=-\bar{p}^{(s)} \delta_{i j}+s \tilde{m}\left(\bar{u}_{i, j}+\bar{u}_{j, i}\right)$,

$\left[n_{i} \bar{t}_{i j}^{(\Delta)}-n_{i} p_{. j}^{(0)} \bar{u}_{j}\right]_{+}=-n_{i} \bar{q}$.

As in (30)-(33), the argument $s$ of arbitrary Laplace-transformed incremental fields will usually be suppressed in the following; for brevity, we refer to such fields simply as 'incremental fields'.

We may use (32) to eliminate $\tilde{t}_{i j}^{(\Delta)}$ from (30) and (33). Observing (13) and (31), we then arrive at the following incremental field equations and boundary condition:

$-\tilde{p}_{, i}^{(\Delta)}+s \tilde{m} \tilde{u}_{i, j j}=0$,

$\tilde{u}_{i, i}=0$,

$\left.\left[n_{i}\left(\bar{p}^{(\Delta)}+p_{, j}^{(0)} \bar{u}_{j}\right)-n_{j} \tilde{s} \tilde{m}_{\left(u_{i, j}\right.}+\tilde{u}_{j, i}\right)\right]_{+}=n_{i} \tilde{q}$.

As an elementary example, we consider perturbations, induced by surface loads, of a plane half-space in a spatially homogeneous gravity field. When applied to planets, this approximation is appropriate only to perturbations whose 'typical' lateral wavelength is short compared with the planet's radius. The symmetry of the half-space suggests to introduce Cartesian coordinates: $\mathbf{X}=(x, y, z)$. We stipulate that $0<x<\infty$ for all $\mathrm{X} \in \mathcal{V}^{(0)}$ such that $x=0$ for all $\mathbf{X} \in \mathscr{A}^{(0)}$. Then, $g_{i}=(g, 0,0)$ must hold, where $g \geq 0$ is a prescribed parameter, and the non-vanishing components of (8) and (10) become

$-p_{. x}^{(0)}+\rho g=0$,

$\left[p^{(0)}\right]_{x=0+}=0$.

Supposing in the following that the perturbing load is $2 \mathrm{D}$, we orient the $y$ axis normal to the strike of the load. It then follows from symmetry considerations that $\tilde{u}_{z}=0, \tilde{p}_{z}^{(\Delta)}=0, \tilde{u}_{i, 2}=0$ and thus also $\tilde{t}_{x z}^{(\Delta)}=\tilde{t}_{y z}^{(j)}=0$. Hence, the non-vanishing scalar components of (32) are

$\tilde{t}_{x x}^{(\Delta)}=-\tilde{p}^{(\Delta)}+2 s \tilde{m} \tilde{u}_{x, x \prime}$

$\bar{t}_{x y}^{(\Delta)}=s \bar{m}\left(\tilde{u}_{x, y}+\bar{u}_{y, x}\right)$,

$\tilde{t}_{y y}^{(\Delta)}=-\tilde{p}^{(\Delta)}+2 s \tilde{m} \bar{u}_{y, y^{\prime}}$

$\tilde{t}_{z z}^{(\Delta)}=-\tilde{p}^{(\Delta)}$.

Similarly, we find for the non-vanishing scalar components of (34) and (35) the relations

$-\tilde{p}_{, x}^{(\lambda)}+s \tilde{m}\left(\bar{u}_{x, x x}+\bar{u}_{x, y y}\right)=0$,

$-\bar{p}_{, y}^{(\Delta)}+s \tilde{m}\left(\bar{u}_{y, x x}+\bar{u}_{y, y y}\right)=0$,

$\vec{u}_{x, x}+\vec{u}_{y, y}=0$.

With (37) and $n_{i}=(1,0,0)$, the scalar components of (36) take the forms

$\left[\bar{u}_{x, y}+\bar{u}_{y, x}\right]_{x=0+}=0$,

$\left[\tilde{p}^{(\Delta)}+\rho g \tilde{u}_{x}-2 s \tilde{m} \tilde{u}_{x, x}\right]_{x=0+}=\tilde{q}$.

Equations (43)-(45) are three simultaneous second-order partial differential equations for $\bar{p}^{(\Delta)}, \tilde{u}_{x}$ and $\bar{u}_{y}$, which must be solved subject to (46) and (47). These equations must be completed by conditions requiring that the incremental fields and their spatial derivatives remain bounded as $x \rightarrow \infty$.

\section{SOLUTION TO THE INCREMENTAL EQUATIONS}

We obtain the general solution to the equations by means of Love's strain function, $\lambda$, defined by (e.g. Malvern, 1969, pp. 552-554)

$\tilde{u}_{x}=\lambda_{, y y}$

$\tilde{u}_{y}=-\lambda, x y$,

$\tilde{p}^{(\Delta)}=-s \tilde{m}\left(\lambda_{, x x}+\lambda_{, y y}\right)_{x}$.

Using (48)-(50), equations (39)-(42) can also be expressed in terms of $\lambda$ :

$\tilde{t}_{x x}^{(\Delta)}=s \tilde{m}\left(\lambda_{. x x}+3 \lambda_{, y y}\right)_{, x}$ 
$\tilde{t}_{x y}^{(\Delta)}=-s \tilde{m}\left(\lambda_{x x}-\lambda_{, y y}\right)_{y,}$

$\tilde{t}_{y y}^{(\Delta)}=s \tilde{m}\left(\lambda_{, x x}-\lambda_{, y, y}\right)_{, x}$

$\tilde{t}_{z z}^{(\Delta)}=s \tilde{m}\left(\lambda_{, x x}+\lambda_{, y y}\right)_{, x}$.

Upon substitution of (48)-(50) into (43)(45), we find that (43) and (45) are identically satisfied. A necessary condition for (44) to be valid is

$\lambda_{, x x x x}+2 \lambda_{, x x y y}+\lambda_{, y y y y}=0$,

which is the biharmonic equation. The general solution to (55) must satisfy the boundary conditions

$\left[\left(\lambda_{, x x}-\lambda_{, y y}\right)_{, y}\right]_{x=0+}=0$,

$\left[\rho g \lambda_{. y y}-s \tilde{m}\left(\lambda_{, x x}+3 \lambda_{. y y}\right)_{x}\right]_{x}=0+=\tilde{q}$,

$\left[\rho g \lambda_{, y y}-s \tilde{m}\left(\lambda_{, x x}+3 \lambda_{, y y}\right)_{, x}\right]_{x=0+}=\tilde{q}$,

which follow from (46) and (47) upon substitution of (48)-(50). An additional requirement is that $\lambda$ and its spatial derivatives remain bounded as $x \rightarrow \infty$.

The equations are solved on the supposition that the incremental fields can be expanded into a Fourier integral. For an arbitrary incremental field component, $\tilde{f}$, and the load pressure, $\bar{q}$, we thus require that

$\tilde{f}(x, y, s)=\int_{-x}^{x} \tilde{F}(x, \xi, s) e^{i \xi y} d \xi$,

$\tilde{q}(y, s)=\int_{-x}^{x} \widetilde{Q}(\xi, s) e^{i \xi y} d \xi$,

where $\bar{F}$ and $\bar{Q}$ are the (complex) spectral densities associated with $\tilde{f}$ and $\tilde{q}$ and $\xi$ is the wave number. Due to their linearity, (48)-(54) then become

$\tilde{U}_{x}=-\xi^{2} \Lambda$,

$\tilde{u}_{y}=-i \xi \Lambda_{, x}$,

$\tilde{P}^{(\Delta)}=-s \tilde{m}\left(\Lambda_{, x x}-\xi^{2} \Lambda\right)_{, x}$

$\tilde{T}_{x x}^{(\Delta)}=s \tilde{m}\left(\Lambda_{, x x}-3 \xi^{2} \Lambda\right)_{, x}$

$\tilde{T}_{x y}^{(\Delta)}=-i \xi \sin \left(\Lambda_{x x}+\xi^{2} \Lambda\right)$,

$\tilde{T}_{y y}^{(\Delta)}=s \tilde{m}\left(\Lambda_{, x x}+\xi^{2} \Lambda\right)_{, x}$

$\tilde{T}_{z z}^{(\Delta)}=\operatorname{s} \tilde{m}\left(\Lambda_{, x x}-\xi^{2} \Lambda\right)_{, x}$

where the arguments $x, \xi$ and $s$ have been suppressed. Similarly, (55)-(57) reduce to

$\Lambda_{, x x x x}-2 \xi^{2} \Lambda_{, x x}+\xi^{4} \Lambda=0$,

$\left[\Lambda_{, x x}+\xi^{2} \Lambda\right]_{x=0+}=0$,

$\left[\xi^{2} \rho g \Lambda+\sin \left(\Lambda_{, x x}-3 \xi^{2} \Lambda\right)_{x}\right]_{x}=0+=-\tilde{Q}$.

(69)
In addition to (69), we require that $\Lambda$ and its spatial derivatives remain bounded as $x \rightarrow \infty$.

For the purposes of the following discussion it will be sufficient to assume $\xi \geq 0$. The solution to (67) satisfying the condition at infinity can then be written in the form

$\Lambda=\frac{1}{\xi^{2}}(A+B \xi x) e^{-\xi x}$,

where $A$ and $B$ are integration constants. Using (70) to substitute for $A$ and its derivatives in (60)-(66), we obtain

$\tilde{U}_{x}=-(A+B \xi x) e^{-\xi x}$,

$\tilde{U}_{y}=i[A-B(1-\xi x)] e^{-\xi x}$,

$\widetilde{P}^{(\Delta)}=-2 \xi s \check{m} B e^{-\xi x}$,

$\tilde{T}_{x x}^{(\Delta)}=2 \xi \sin (A+B \xi x) e^{-\xi x}$,

$\widetilde{T}_{x y}^{(\Delta)}=-2 i \xi s \tilde{m}[A-B(1-\xi x)] e^{-\xi x}$,

$\tilde{T}_{y y}^{(\Delta)}=-2 \xi s \tilde{m}[A-B(2-\xi x)] e^{-\xi x}$,

$\tilde{T}_{z z}^{(1)}=2 \xi s \tilde{m} B e^{-\xi x}$.

The constants $A$ and $B$ can be determined by substitution of $(70)$ and its derivatives into (68) and (69). We get

$A=B=-\frac{\tilde{Q}}{2 \xi \operatorname{sim}+\rho g}$,

whence (71)-(77) take the forms

$\tilde{U}_{x}=\frac{\widetilde{Q}}{2 \xi s \tilde{m}+\rho g}(1+\xi x) e^{-\xi x}$,

$\widetilde{U}_{y}=-i \frac{\tilde{Q}}{2 \xi \mathrm{s} \tilde{m}+\rho g} \xi x e^{-\xi x}$,

$\widetilde{p}^{(\Delta)}=\frac{2 \xi s \tilde{m} \tilde{Q}}{2 \xi s \tilde{m}+\rho g} e^{-\xi x}$,

$\tilde{T}_{x x}^{(\Delta)}=-\frac{2 \xi s \tilde{m} \tilde{Q}}{2 \xi s \tilde{m}+\rho g}(1+\xi x) e^{-\xi x}$,

$\widetilde{T}_{x y}^{(\Delta)}=i \frac{2 \xi s \tilde{m} \tilde{Q}}{2 \xi s \tilde{m}+\rho g} \xi x e^{-\xi x}$,

$\bar{T}_{y y}^{(\Delta)}=-\frac{2 \xi s \tilde{m} \widetilde{Q}}{2 \xi s \tilde{m}+\rho g}(1-\xi x) e^{-\xi x}$,

$\tilde{T}_{z z}^{(\Delta)}=-\frac{2 \xi \tilde{s} \tilde{m} \tilde{Q}}{2 \xi s \tilde{m}+\rho g} e^{-\xi x}$.

A useful incremental field quantity to consider is the maximum shear stress occurring in the half-space. Since the maximum shear stress can be related to the difference between the largest and smallest principal stresses, we must first determine the principal stresses. With $\tilde{t}_{z z}^{(\Delta)}=-\tilde{p}^{(\Delta)}$ as one of the principal stresses, the other two are obtained from the characteristic equation $\operatorname{det}\left[\begin{array}{ll}\tilde{t}_{x x}^{(\Delta)}-\tilde{t}^{(\Delta)} & \tilde{t}_{x y}^{(\Delta)} \\ \tilde{t}_{x y}^{(\Delta)} & \tilde{t}_{y y}^{(\Delta)}-\tilde{f}^{(\Delta)}\end{array}\right]=0$.

Upon expansion of the determinant and use of (50), (51) and (53), the principal stresses are found to be

$\tilde{t}_{1,2}^{(\Delta)}=-\tilde{p}^{(\Delta)} \pm\left[\frac{\left(\tilde{t}_{x x}^{(\Delta)}-\tilde{t}_{y y}^{(\Delta)}\right)^{2}}{4}+\left(\tilde{t}_{x y}^{(\Delta)}\right)^{2}\right]^{1}$.

Since $\vec{t}_{1}^{(\Delta)}$ and $\vec{t}_{2}^{(1)}$ are the largest and smallest principal stresses, respectively, the maximum shear stress, $i_{s}$ is given by

$\tilde{t}_{s}=\frac{1}{2}\left(\tilde{t}_{1}^{(\Delta)}-\tilde{t}_{2}^{(\Delta)}\right)$.

Note that we have not added a superscript to the symbol $\tilde{t}_{\mathrm{s}}$. This is in accordance with $(20)$, which shows that no distinction between material and local increments is required if shear stress components or differences between normal stress components are considered. Substitution of (87) into (88) gives

$\bar{t}_{s}=\frac{1}{2}\left[\left(\tilde{t}_{x x}^{(\Delta)}-\tilde{t}_{y y}^{(\Delta)}\right)^{2}+4\left(\tilde{t}_{x y}^{(\Delta)}\right)^{2}\right]^{t}$.

If we put

$\bar{t}_{\mathrm{s}}=\int_{-\pi}^{x} \tilde{T}_{s} d \xi$

and equate (89) with (90), we find, using (58) and (82)-(84), the relation

$\widetilde{T}_{s}=(1+i) \frac{2 \xi s \tilde{m} \bar{Q}}{2 \xi s \tilde{m}+\rho g} \xi x e^{-\xi x}$.

Note that, according to $(90)$ and $(91), \bar{t}_{s}$ is independent of $y$.

To proceed beyond (79)-(85) and (91) we must specify $\tilde{m}$ and $\tilde{Q}$. As a simple example, we consider the shear relaxation function for Maxwellian viscoelasticity (e.g. Christensen, 1982, pp. 16-20):

$m=\mu H(t) e^{-\alpha !}$.

Note that $m$ is determined by two parameters: the inverse Maxwell time, $\boldsymbol{\alpha}$, and the shear modulus, $\mu$. We further consider an 'instantaneous' loading event:

$Q=Q^{\prime} H(t)$.

In view of (28), the Laplace transforms of (92) and (93) are

$\widetilde{m}=\frac{\mu}{s+\alpha}$, 


$$
\tilde{Q}=\frac{Q^{\prime}}{s}
$$

From (94) and (95), we get

$$
\frac{\widetilde{Q}}{2 \xi s \tilde{m}+\rho g}=\frac{Q^{\prime}}{2 \xi \mu+\rho g}\left[\frac{1}{s}+\frac{2 \xi \mu}{\rho g}\left(\frac{1}{s}-\frac{1}{s+\beta}\right)\right]
$$

$\frac{2 \xi s \tilde{m} \bar{Q}}{2 \xi s \tilde{m}+\rho g}=\frac{2 \xi \mu Q^{\prime}}{2 \xi \mu+\rho g} \frac{1}{s+\beta^{\prime}}$

where

$\beta=\frac{\rho g \alpha}{2 \xi \mu+\rho g}$.

Upon substitution of (96) and (97) and subsequent inverse Laplace transformation using (28), equations (79)-(85) and (91) become

$$
\begin{aligned}
U_{x}= & \frac{Q^{\prime} H(t)}{2 \xi \mu+\rho g} \\
& \times\left[1+\frac{2 \xi \mu}{\rho g}\left(1-e^{-\beta t}\right)\right](1+\xi x) e^{-\xi x},(99) \\
U_{y}= & -i \frac{Q^{\prime} H(t)}{2 \xi \mu+\rho g} \\
& \times\left[1+\frac{2 \xi \mu}{\rho g}\left(1-e^{-\beta t}\right)\right] \xi x e^{-\xi x}, \quad(100) \\
P^{(\Delta)}= & \frac{2 \xi \mu Q^{\prime} H(t)}{2 \xi \mu+\rho g} e^{-\beta t} e^{-\xi x}, \\
T_{x x}^{(\Delta)}= & -\frac{2 \xi \mu Q^{\prime} H(t)}{2 \xi \mu+\rho g} e^{-\beta t}(1+\xi x) e^{-\xi x},(102) \\
T_{x y}^{(\Delta)}= & i \frac{2 \xi \mu Q^{\prime} H(t)}{2 \xi \mu+\rho g} e^{-\beta t} \xi x e^{-\xi x}, \\
T_{y y}^{(\Delta)}= & -\frac{2 \xi \mu Q^{\prime} H(t)}{2 \xi \mu+\rho g} e^{-\beta t}(1-\xi x) e^{-\xi x},(104) \\
T_{z z}^{(\Delta)}= & -\frac{2 \xi \mu Q^{\prime} H(t)}{2 \xi \mu+\rho g} e^{-\beta t} e^{-\xi x}, \\
T_{s}= & (1+i) \frac{2 \xi \mu Q^{\prime} H(t)}{2 \xi \mu+\rho g} e^{-\beta t} \xi x e^{-\xi x} .
\end{aligned}
$$

In view of (99)-(106), $\boldsymbol{\beta}$ will be referred to as the inverse relaxation time.

\section{DISCUSSION OF THE SOLUTION}

For the discussion of the solution, we consider the surface of the half-space, $x=0+$. It then follows from (99)-(106) that $U_{y}=0, T_{x x}^{(\Delta)}=T_{y y}^{(\Delta)}=T_{z 2}^{(\Delta)}=-P^{(\Delta)}$ and $T_{x y}^{(\Delta)}=T_{s}=0$. Hence, the nonvanishing independent surface components are

$$
\left[U_{x}\right]_{x=0+}=\frac{Q^{\prime} H(t)}{2 \xi \mu+\rho g}\left[1+\frac{2 \xi \mu}{\rho g}\left(1-e^{-\beta t}\right)\right]
$$

$\left[P^{(\Delta)}\right]_{x=0+}=\frac{2 \xi \mu Q^{\prime} H(t)}{2 \xi \mu+\rho g} e^{-\beta t}$.

We also consider the depth, $x_{m}$, where $T_{s}$ assumes a maximum. Since

$\frac{d}{d(\xi x)}\left(\xi x e^{-\xi x}\right)=(1-\xi x) e^{-\xi x}$,

we obtain

$x_{m}=\frac{1}{\xi}$.

Substitution of (110) into (106) then gives

$\left[T_{s}\right]_{x=x_{m}}=\frac{1+i}{e} \frac{2 \xi \mu Q^{\prime} H(t)}{2 \xi \mu+\rho g} e^{-\beta t}$.

The short-time limits of (107), (108) and (111) are

$$
\begin{aligned}
& {\left[U_{x}\right]_{x=0+, t=0+}=\frac{Q^{\prime}}{2 \xi \mu+\rho g^{\prime}},} \\
& {\left[P^{(\Delta)}\right]_{x=0+, t=0+}=\frac{2 \xi \mu Q^{\prime}}{2 \xi \mu+\rho g},} \\
& {\left[T_{s}\right]_{x=x_{m}, t=0+}=\frac{1+i}{e} \frac{2 \xi \mu Q^{\prime}}{2 \xi \mu+\rho g} .}
\end{aligned}
$$

Equations (112)-(114) apply to elastostatic equilibrium, governed by the shear modulus $\mu$, in a half-space subject to the hydrostatic initial stress gradient pg (e.g. Wolf, 1985a, 1985b). Using (98), the long-time limits of (107), (108) and (111) are found to be

$$
\begin{aligned}
& {\left[U_{x}\right]_{x=0+, t \rightarrow \infty}=\frac{Q^{\prime}}{\rho g},} \\
& {\left[P^{(\Delta)}\right]_{x=0+, t \rightarrow \infty}=0,} \\
& {\left[T_{s}\right]_{x=x_{m}, t \rightarrow \infty}=0 .}
\end{aligned}
$$

Equations (115) describe hydrostatic equilibrium in a half-space subject to the hydrostatic initial stress gradient $\rho g$. The transition from the instantaneous elastostatic to the final hydrostatic equilibrium state is seen from (107), (108) and (111) to be exponential in time, where the inverse relaxation time, $\beta$, is related to the parameters of the halfspace by (98).

If $2 \xi \mu /(\rho g) \ll 1$, equations $(98)$, (107), (108) and (111) become, correct to the first order in the small quantity,

$\beta \simeq \alpha\left(1-\frac{2 \xi \mu}{\rho g}\right)$,

$\left[U_{x}\right]_{x=0+} \approx \frac{Q^{\prime} H(t)}{\rho g}\left(1-\frac{2 \xi \mu}{\rho g} e^{-\beta t}\right)$,

$\left[P^{(\Delta)}\right]_{x=0+} \simeq \frac{2 \xi \mu Q^{\prime} H(t)}{\rho g} e^{-\beta t}$,
$\left[T_{s}\right]_{x=x_{m}}=\frac{1+i}{e} \frac{2 \xi \mu Q^{\prime} H(t)}{\rho g} e^{-\beta x}$.

A comparison between (98) and (118) shows that $\beta$ is now close to its largest value, $\alpha$, i.e. the relaxation proceeds rapidly. According to (119)-(121), however, the relaxation is insignificant in amplitude and vanishes for $\xi \mu=0$. For perturbations of sufficiently long wavelength, the differences between the elastostatic and hydrostatic equilibrium states may therefore be ignored.

If $\rho g /(2 \xi \mu) \ll 1$ and $\beta t \ll 1$, equations (98), (107), (108) and (111) become, correct to the first order in the small quantities,

$\beta=\frac{\rho g \alpha}{2 \xi \mu}$,

$\left[U_{x}\right]_{x=0+} \approx \frac{Q^{\prime} H(t)}{2 \xi \mu}\left(1-\frac{\rho g}{2 \xi \mu}+\alpha t\right)$,

$\left[P^{(\Delta)}\right]_{x=0+} \approx Q^{\prime} H(t)\left(1-\frac{\rho g}{2 \xi \mu}-\beta t\right),(12$

$\left[T_{s}\right]_{x=x_{m}}=\frac{1+i}{e} Q^{\prime} H(t)\left(1-\frac{\rho g}{2 \xi \mu}-\beta t\right)$.

Equation (122) is the expression for the inverse relaxation time for Newtonianviscous perturbations, controlled by the shear viscosity modulus $\mu / \alpha$, of a halfspace subject to the hydrostatic initial stress gradient $\rho g$ (Haskell, 1935, 1936; Ranalli, 1987, pp. 192-199).

If $\rho g /(2 \xi \mu)=0$, equations (122)-(125) further reduce to

$\beta=0$,

$\left[U_{x}\right]_{x=0+}=\frac{Q^{\prime} H(t)}{2 \xi \mu}(1+\alpha t)$,

$\left[P^{(\Delta)}\right]_{x=0+}=Q^{\prime} H(t)$,

$\left[T_{s}\right]_{x=x_{t t}}=\frac{1+i}{e} Q^{\prime} H(t)$.

For $t=0+$, equations (127)-(129) apply to elastostatic equilibrium in an initially unstressed half-space (e.g. Jeffreys, 1976, pp. 265-267). Equation (126) shows that the subsequent relaxation proceeds infinitely slow. On the other hand, it follows with (115) that

$\left[U_{x}\right]_{x}=0+, 1 \rightarrow \infty \rightarrow \infty$,

where the instability is due to the absence of the gravitational force necessary to balance the load in the final hydrostatic state. 
More practically, we may use the condition $\rho g /(2 \xi \mu) \leq 10^{-2}$ to estimate the minimum value of $\xi$ in order that effects due to the hydrostatic initial.stress may be neglected. Considering the Earth as an example and taking $\rho \simeq 10^{3} \mathrm{~kg} \mathrm{~m}^{-3}$, $g \simeq 10 \mathrm{~m} \mathrm{~s}^{-2}$ and $\mu \simeq 10^{11} \mathrm{~Pa}$ as values typical of the Earth, we obtain $\xi=10^{-5}$ $\mathrm{m}^{-1}$ as the minimum wave number. This approximately corresponds to a maximum wavelength of $10^{6} \mathrm{~m}$. Within the limits of the half-space approximation, the influence of the initial stress is therefore negligible as far as elastic perturbations are concerned. However, if $\mu \ll 10^{11} \mathrm{~Pa}$, the initial stress becomes noticeable at much shorter wavelengths. This condition in particular applies to viscoelastic perturbations, where the 'effective' shear modulus, $s \tilde{m}$, may become arbitrarily small.

Additional insight is gained by considering also $P^{(8)}$. In view of (19), (37) and (58), we have in particular

$\left[P^{(\delta)}\right]_{x=0+}=\left[P^{(\lrcorner)}\right]_{x=0+}+\rho g\left[U_{x}\right]_{x=0+}$

Upon substitution of (107) and (108), this becomes

$\left[P^{(\delta)}\right]_{x=0+}=Q^{\prime} H(t)$.

Since it is readily shown that $\left[T_{x x}^{(\delta)}\right]_{x=0+}$ $=-\left[P^{(\delta)}\right]_{x}=0+$, equation (132) simply expresses the balance required by (18) between the material incremental stress component normal to the surface of the half-space and the incremental load pressure.

In view of (116), it follows from (131) that

$\left[P^{(\delta)}\right]_{x=0+, t \rightarrow \infty}=\rho g\left[U_{x}\right]_{x}=0+, t \rightarrow \infty .(133$

In the final hydrostatic equilibrium state, the material incremental pressure is thus completely maintained by the advective incremental pressure associated with the displacement component in the direction of the initial pressure gradient. Assuming that (116) remains valid for $\rho g \rightarrow 0$, equation (133) also applies in this case and we require

$$
\lim _{\substack{\rho g \rightarrow 0 \\\left[U_{\mathrm{x}}\right]_{x=0+. t \rightarrow \infty} \rightarrow \infty}} \rho g\left[U_{x}\right]_{x=0+, t \rightarrow \infty}=Q^{\prime} .
$$

Together, (133) and (134) ensure that the incremental boundary condition given by (132) is satisfied in the final hydrostatic state even in the absence of initial stress. This illustrates the physical significance of the singularity in the solution for the displacement noted in (130) from a different point of view.

\section{ACKNOWLEDGEMENTS}

This investigation is an extended version of a lecture given by the author at the Institute of Geophysics, Uppsala University, when he was a visiting scientist at the Department of Geodesy. The visit was financially supported by the Swedish Natural Science Research Council (NFR).

\section{REFERENCES}

Biot M.A. (1959) The influence of gravity on the folding of a layered viscoelastic medium under compression, J. Franklin Inst., 267, 211-228.

Biot M.A. (1965) Mechanics of Incremental Deformations. Wiley, New York.

Boussinesq J. (1885) Application des potentiels à l'étude de l'équilibre et du mouvement des solides élastiques, principalement au calcul des déformations et des pressions que produisent, dans ces solides, des efforts quelconques exercés sur une petite partie de leur surface ou de leur intérieur. GauthierVillars, Paris.

Cathles L.M. (1975) The Viscosity of the Earth's Mantle. Princeton University Press, Princeton, NJ.

Cerruti V. (1882) Ricerche intorno all' equilibrio $\mathrm{de}^{\prime}$ corpi elastici isotropi, Reale Accad. Linc. Roma, Ser. 3A, 13, 81122.

Christensen R.M. (1982) Theory of Viscoelasticity, 2nd edn. Academic Press, New York.

Dahlen F.A. (1974) On the static deformation of an earth model with a fluid core, Geophys. J. R. astr. Soc., 36, 461-485.

Farrell W.E. (1972) Deformation of the earth by surface loads, Rev. Geophys. Space Phys., 10, 761-797.

Grafarend E.W. (1982) Six lectures on geodesy and global geodynamics, Mitt. Geodät. Inst. Techn. Univ. Graz, 41, 531-685.

Harding J.W. and Sneddon I.N. (1945) The elastic stresses produced by the indentation of the plane surface of a semi-infinite elastic solid by a rigid punch, Proc. Cambr. Phil. Soc., 41, 16-26.

Haskell N.A. (1935) The motion of a viscous fluid under a surface load,
Physics, 6, 265-269.

Haskell N.A. (1936) The motion of a viscous fluid under a surface load II, Physics, 7, 56-61.

Jeffreys H. (1976) The Earth: Its Origin, History and Physical Constitution, 6th edn. Cambridge University Press, Cambridge.

Lamb H. (1902) On Boussinesq's problem, Proc. London Math. Soc. , 34, 276-284.

Lamé G. and Clapeyron E. (1831) Mémoire sur l'équilibre intérieur des corps solides homogènes, Crelle's I. Reine Angew. Math., 7, 381-413.

LePage W.R. (1961) Complex Variables and the Laplace Transform for Engineers. McGraw-Hill, New York.

Love A.E.H. (1929) The stress produced in a semi-infinite solid by pressure on part of the boundary, Phil. Trans. R. Soc. A, 228, 377-420.

Malvern, L.E. (1969) Introduction to the Mechanics of a Continuous Medium. Prentice-Hall, Englewood Cliffs.

Nakiboglu S.M. and Lambeck K. (1982) A study of the earth's response to surface loading with application to Lake Bonneville, Geophys. J. R. astr. Soc., 70, 577-620.

Peltier W.R. (1974) The impulse response of a Maxwell earth, Rev. Geophys. Space Phys., 12, 649-669.

Ranalli G. (1987) Rheology of the Earth: Deformation and Flow Processes in Geophysics and Geodynamics. Allen and Unwin, Boston.

Sneddon I.N. (1946) The elastic stresses produced in a thick plate by the application of pressure to its free surfaces, Proc. Cambr. Phil. Soc., 42, 260-271.

Terazawa K. (1916) On the elastic equilibrium of a semi-infinite solid under given boundary conditions, with some applications, I. Coll. Sci. Tokyo, 37/7, 1-64.

Wolf D. (1985a) Thick-plate flexure reexamined, Geophys. I. R. astr. Soc., 80, 265-273.

Wolf D. (1985b) On Boussinesq's problem for Maxwell continua subject to an external gravity field, Geophys. J. R. astr. Soc., 80, 275-279.

Wolf D. (1985c) The normal modes of a uniform, compressible Maxwell halfspace, J. Geophys., 56, 100-105.

Wolf D. (1991) Viscoelastodynamics of a stratified, compressible planet: incremental field equations and short- and long-time asymptotes, Geophys. I. Int., 104, 401-417. 


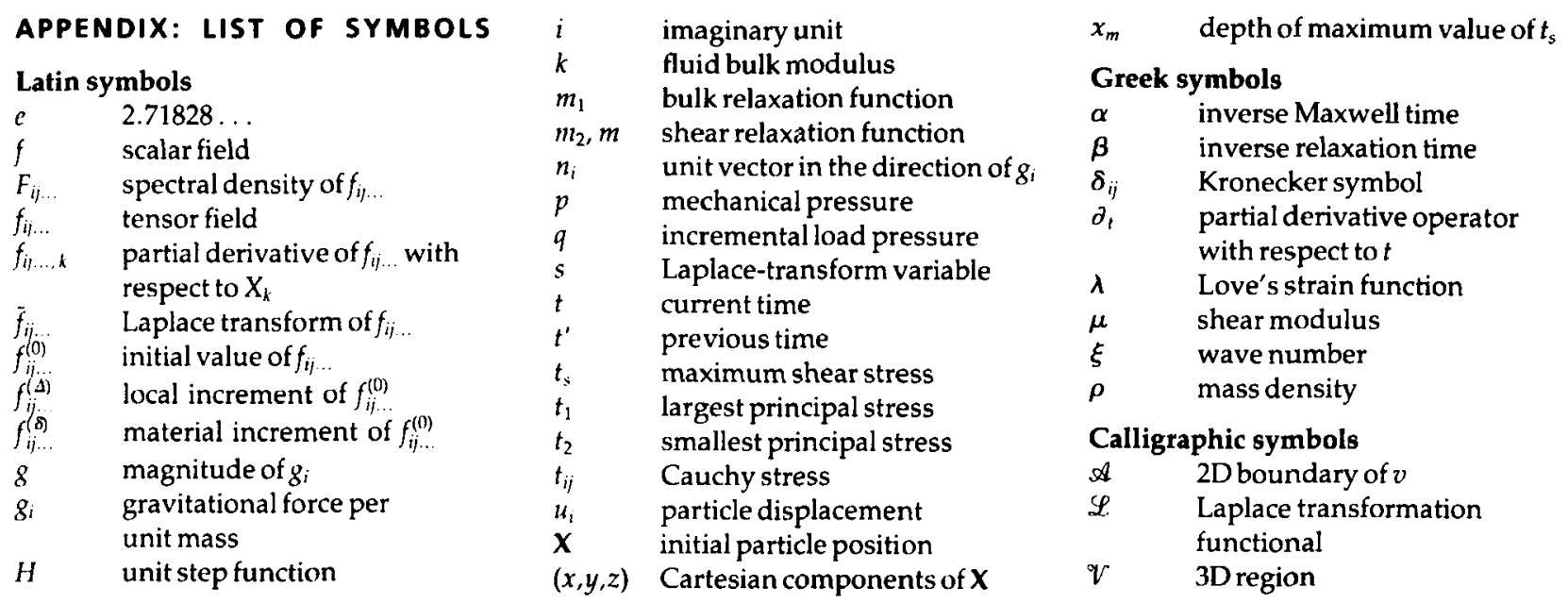

